\title{
Nanocellulose and chitosan based films as low cost, green piezoelectric materials
}

Aleksi Hänninen $^{\mathrm{a}}$, Essi Sarlin ${ }^{\mathrm{b}}$, Inari Lyyra ${ }^{\mathrm{a}}$, Timo Salpavaara ${ }^{\mathrm{a}}$, Minna Kellomäki ${ }^{\mathrm{a}, \mathrm{c}}$, Sampo

Tuukkanen $^{\mathrm{a}}$

${ }^{a}$ BioMediTech Institute and Faculty of Biomedical Sciences and Engineering, Tampere University of Technology, Korkeakoulunkatu 10, Tampere 33720, Finland

${ }^{\mathrm{b}}$ Faculty of Engineering Sciences, Tampere University of Technology, Korkeakoulunkatu 10, Tampere 33720, Finland

'BioMediTech Institute and Faculty of Medicine and Life Sciences, University of Tampere, Arvo Ylpön katu 34, Tampere 33520, Finland

\begin{abstract}
Nanocellulose and chitosan have recently started to get attention as environmentally friendly piezoelectric materials for sensor and energy harvesting applications. Conversely, current commercially available flexible piezoelectric films made of for example polyvinylidene difluoride (PVDF) are relatively expensive and made from non-renewable materials. We measured the piezoelectric responses $(2-8 \mathrm{pC} / \mathrm{N})$ for solvent casted films based on nanocellulose, microcrystalline chitosan and their blends. In addition, the tensile properties of the piezoelectric films were characterized to find out if chitosan could be used to enhance the flexibility of the brittle nanocellulose films. Based on the results, plain chitosan is an interesting piezoelectric material itself. In addition, blending nanocellulose and chitosan could be a potential method for tailoring the properties of solvent casted low cost, green piezoelectric films.
\end{abstract}

\section{Introduction}

Cellulose is found in multiple sources including seeds, wood, vegetables and fruits, making it the most abundant polymer in the world (Leite, Zanon, \& Menegalli, 2017). Cellulose fibrils can be isolated from the surrounding lignin and hemicellulose matrices by a chemical pulping treatment, where fragmented lignin and hemicellulose are solubilized (Ditzel, Prestes, Carvalho, Demiate, \& Pinheiro, 2017). Various processing routes have been developed to obtain nanocellulose structures like cellulose nanofibrils (CNF) or cellulose nanocrystals (CNC) where the amorphous cellulose parts are mostly removed (Xu et al., 2013). The proportions of the crystalline and amorphous phases vary among different cellulose sources, which has an effect on the resulting nanocellulose characteristics (Morais et al., 2013).

Based on production volumes the second most abundant polymer after cellulose is chitin, whose most important sources commercially are crab and shrimp shells (Rinaudo, 2006). In addition to crustaceans, it is found for example in insects, mollusks and cell walls of fungi (Grifoll-Romero, Pascual, Aragunde, Biarnés, \& Planas, 2018). Deacetylating chitin enzymatically or under alkaline conditions yields chitosan, which is soluble in aqueous acidic 
media. Depending on the chitin source, the degree of deacetylation should reach approximately $50 \%$ before this solubility is achieved. (Rinaudo, 2006) In nature, chitosan is reportedly produced by only some fungi (Grifoll-Romero et al., 2018).

Nanocellulose and chitosan have been studied for numerous applications due to their simple water based solution processing, renewable raw material sources, low cost, biocompatibility and biodegradability (Bharimalla, Deshmukh, Vigneshwaran, Patil, \& Prasad, 2017; Fernandes et al., 2010; Kumar, 2000; No, Meyers, Prinyawiwatkul, \& Xu, 2007). Both biopolymers are known to degrade via enzymatic routes (Tomihata \& Ikada, 1997)(Singh et al., 2016), which makes them promising alternatives for petroleum-based materials.

New potential applications for nanocellulose and chitosan are being sought in many areas, including the emerging field of biodegradable energy harvesters and sensors. Such applications are based on the piezoelectricity of these materials, which means they have the ability to develop electrical charges under mechanical stress (Safari \& Janas, 1997). As an example, the increased amount of smart devices in the Internet of Things (IoT) has raised concerns about the associated waste and emission generation (Maksimovic, 2017). Using biodegradable piezoelectric membranes for local energy production could power the IoT in an environmentally and economically sound fashion compared to non-degradable materials or batteries.

A large amount of piezoelectric materials are known, with a common requirement of having a non-centrosymmetric crystal structure (Randall, Kelnberger, Yang, Eitel, \& Shrout, 2005). The piezoelectric properties of CNF and CNC have raised wide interest only during the past few years (Csoka et al., 2012; Frka-Petesic, Jean, \& Heux, 2014; Rajala et al., 2016; Rajala, Vuoriluoto, Rojas, Franssila, \& Tuukkanen, 2015; Tuukkanen \& Rajala, 2015), although the piezoelectricity of wood cellulose has been known for decades (Fukada, 1955).

Correspondingly, the piezoelectricity of chitin has been reported in the 1970s (Fukada \& Sasaki, 1975), but literature concerning the piezoelectric sensitivity of chitosan is scarce. Piezoelectric sensitivity values of chitosan have been reported in our preliminary study (Hänninen, Rajala, Salpavaara, Kellomäki, \& Tuukkanen, 2016) as well as in a recent study where the usage of chitosan in piezoelectric vibration sensors was reported (Praveen, Murugan, \& Jayakumar, 2017).

In the present study, we characterized the properties of nanocellulose, microcrystalline chitosan and their blend films. The films were prepared by solvent casting and their structural, mechanical and piezoelectric properties were studied by microscopy, tensile test and piezoelectric measurements. This work was motivated by the limited handling durability of plain nanocellulose films. Our hypothesis was that forming nanocellulose-chitosan blends would enhance the durability of the films.

\section{Materials \& Methods}

\subsection{Film preparation}

The films were solvent casted from nanocellulose and chitosan solutions prepared as follows: cellulose nanofiber (CNF) hydrogel dispersion (1.4 wt-\%, pH 6.6) was produced by 
mechanically homogenizing bleached birch cellulose mass. The process is explained in detail in (Rajala et al., 2016). Acidic cellulose nanocrystal (CNC) dispersion (1.4 wt-\%, pH 2.9) was obtained by chemically hydrolyzing CNF in sulfuric acid. The dimensions of CNF and CNC were measured by atomic force microscope (AFM Veeco Dimension D5000). The diameters of CNF and CNC ranged between 5-100 nm and 8-9 nm, respectively. The length of the CNF exceeded $10 \mu \mathrm{m}$, whereas the CNC whisker length was 100-200 nm.

A 1 wt-\% water based chitosan solution ( $\mathrm{pH}$ 3.1) was prepared using medical grade off-white microcrystalline chitosan flakes (Protasan UP B 90/500; FMC Health and Nutrition, Norway) with a degree of deacetylation of $91 \%$ and a molecular weight of $460000 \mathrm{~g} / \mathrm{mol}$. Chitosan was weighed into distilled water $\left(\mathrm{dH}_{2} \mathrm{O}\right)$, after which $90 \%$ lactic acid (VWR Chemicals, Belgium) was added to dissolve the chitosan. The $\mathrm{dH}_{2} \mathrm{O}$-lactic acid ratio was 100:1. All the materials and chemicals were used without further purification.

A total of $20 \mathrm{ml}$ of solution (recipes presented in Table 1) per film was casted onto a Petri dish and allowed to dry at room temperature. Prior to casting, the blend films were mixed until the solution appeared uniform. After drying, the residual acids in the films were neutralized with $1 \mathrm{M} \mathrm{NaOH}$. The films were then peeled off from the dishes and washed with $\mathrm{dH}_{2} \mathrm{O}$ until the $\mathrm{pH}$ of the washing water was 7. The neutralized freestanding films were redried under tension by placing them on top of glass jars using rubber bands. The plain CNF films were noticed to tear easily when handling them wet; they were thus detached from the Petri dishes without the $\mathrm{NaOH}$ treatment and the subsequent drying phase.

Table 1. The recipes for the solvent casted films.

\begin{tabular}{ll} 
Solution volumes & Weight proportion \\
\hline $\mathbf{2 0} \mathbf{~ m l ~ c h i t o s a n ~ s o l u t i o n ~}$ & $100 \%$ chitosan \\
$\mathbf{1 0} \mathbf{~ m l}$ CNC dispersion + $\mathbf{1 0} \mathbf{~ m l}$ chitosan & $58 \%$ cellulose nanocrystals; 42\% chitosan \\
$\mathbf{1 0} \mathbf{~ m l ~ C N F}$ hydrogel dispersion + $\mathbf{1 0} \mathbf{~ m l ~ c h i t o s a n ~}$ & $58 \%$ cellulose nanofibers; 42\% chitosan \\
$\mathbf{2 0} \mathbf{~ m l ~ C N F}$ hydrogel dispersion & $100 \%$ cellulose nanofibers \\
\hline
\end{tabular}

\subsection{Structural evaluation of the films}

The structure of the piezoelectric films was characterized by optical microscopy, scanning electron microscopy (SEM) and by measuring their thickness and density values. The optical microscopy images were taken with an Olympus light microscope (Olympus BH-2, Olympus Optical Co., Tokyo, Japan). Both sides of the films were imaged using a 10x magnification. The SEM (Zeiss ULTRAplus, Germany) images were taken after coating the samples with a thin carbon layer to provide electrical conductivity prior to the imaging. Cross sectional images were obtained by tearing the outer side of the films and gluing the samples to holders with conductive carbon cement.

Thermogravimetric analysis (TGA) was performed using Netzsch TG 209 Tarsus equipment. Measurements were conducted in $\mathrm{N}_{2}$ atmosphere from 25 to $900{ }^{\circ} \mathrm{C}$ with a heating rate of 10 ${ }^{\circ} \mathrm{C} / \mathrm{min}$. A few milligrams from each film was heated. 
To measure the thickness and the density of the films, seven round pieces (diameter $8 \mathrm{~mm}$ ) were cut out from each film type using a metallic punch cutter. The thickness and mass of each piece were measured using a micrometer and a microbalance, respectively. The average film thickness and apparent film density were then calculated. An even film thickness of the round pieces was assumed in the density calculations.

\subsection{Tensile testing}

The films were prepared for tensile testing by punching with a dog-bone shaped cutter; the distance between the shoulders was $20 \mathrm{~mm}$ and the width $5 \mathrm{~mm}$. All samples were visually inspected against cracks. The tensile properties were measured from dry samples using Instron 4411 materials testing machine (Instron Ltd., High Wycombe, England) in ambient conditions. Distance between the pneumatic grips was $20 \mathrm{~mm}$ and a $500 \mathrm{~N}$ load cell were used. The crosshead speed was set to $30 \mathrm{~mm} / \mathrm{min}$. Three parallel samples of each material were measured. The Young's Modulus, elongation at break and the tensile strength of the films were calculated.

\subsection{Sensor assembly and piezoelectric measurements}

Electrodes with a $15 \mathrm{~mm}$ diameter were fabricated on $125 \mu \mathrm{m}$ polyethylene terephthalate (PET, Melinex ST506) substrates. A $100 \mathrm{~nm}$ thick silver layer was e-beam evaporated through a laser-cut stencil shadow mask. The measured films were then placed between two electrodes. Crimp connectors were used to provide the connections for the piezoelectric sensitivity measurements. The sensor assembly is illustrated in (Rajala et al., 2016).

The Brüel \& Kjaer Mini-Shaker Type 4810 generated a sinusoidal excitation force with an amplitude of $1.3 \mathrm{~N}$ for the sensor sensitivity measurements. A dynamic force sensor (PCB Piezotronics, model 209C02) was used as a reference sensor for the excitation force. A static pre-compression force of $3 \mathrm{~N}$ was used to keep the sample in place and to prevent the piston from jumping off the surface during the measurement. The pre-compression force was measured using a load cell (Measurement Specialties Inc., model ELFS-T3E-20L).

The generated charges were measured with the method described in (Rajala et al., 2016). The charges were divided by the dynamic force to obtain the piezoelectric sensitivities (unit $\mathrm{pC} / \mathrm{N})$. Both sample sides were measured to see if the drying phase of the films results in differences between the two sides. Ten parallel measurements per side were performed and two different films per material were tested, resulting in 20 measurements per side for each material.

\section{Results and discussion}

\subsection{Structure of the films}

The optical microscopy images (Fig. 1) showed a rough surface structure in the blend films. The chitosan film was very smooth. During film preparation, the bottom surface lied against the smooth glass dish. As illustrated by the SEM images, this led to smoother bottom surface of the blend films compared to their top surface. The white patterns seen on the CNF bottom surface were interpreted as fibers sticking out, possibly as a result of film detachment. The CNC-chitosan film bottom was slightly wrinkled, but not as strongly as the top side. 

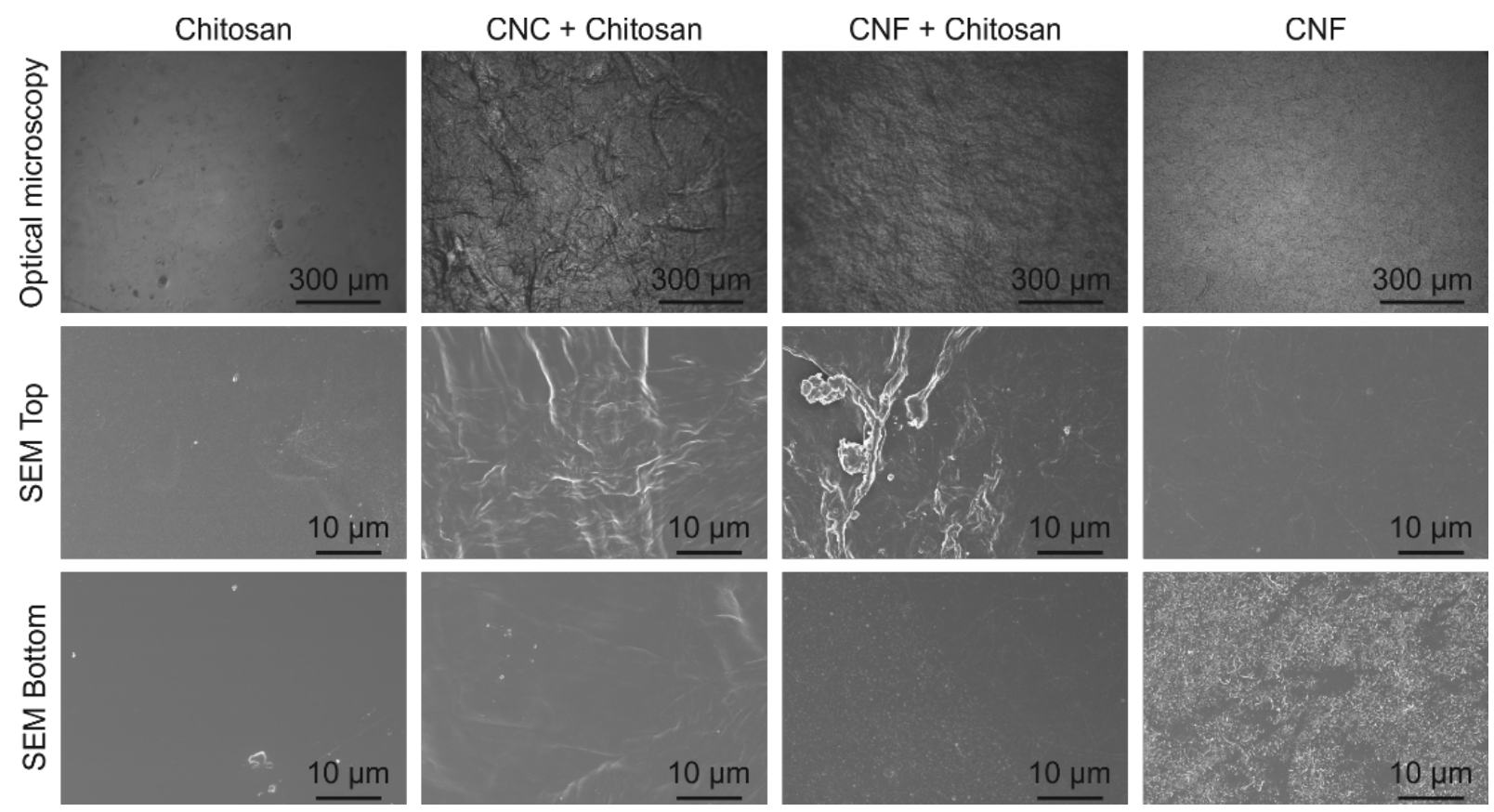

Fig. 1. Optical microscopy and SEM images showing structural features of the piezoelectric films.

The cross sectional SEM images (Fig. 2) indicate that the structure of the chitosan film was homogenous. The blend films as well as the plain CNF showed a stratified structure: in each layer, the fibers were located in the same plane. The structure of the blend films was more irregular than that of the plain chitosan and CNF films. Still, both blend types indicated good dispersion between the matrix and the filler unlike in our preliminary study (Hänninen et al., 2016), where separate chitosan and nanocellulose layers were observed. This might have resulted from different drying conditions. Previous reports have indicated that nanocellulose forms strong interactions with chitosan (Sundaram, Pant, Goudie, Mani, \& Handa, 2016; Tomé et al., 2013), which is in agreement with the current result. 

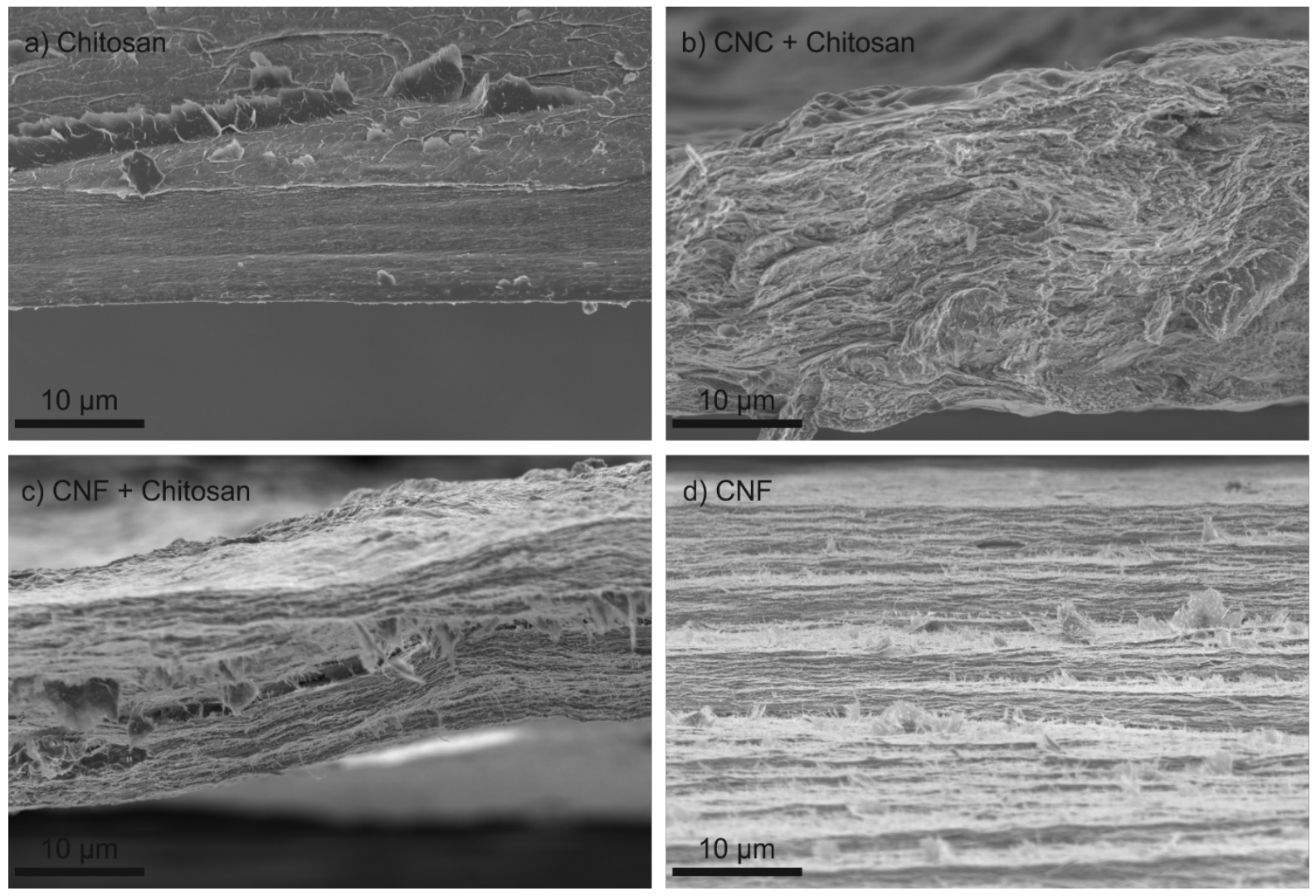

Fig. 2. Cross-sectional SEM images of a) chitosan b) CNC-chitosan c) CNF-chitosan and d) plain CNF films.

Fig. 3 presents the mean thickness and density of the piezoelectric films. The CNC-chitosan $(41 \mu \mathrm{m})$ and CNF-chitosan $(40 \mu \mathrm{m})$ films were approximately twice as thick as the plain chitosan film $(20 \mu \mathrm{m})$. The highly viscous CNF hydrogel was challenging to cast evenly onto the dish, resulting in non-uniform CNF film thicknesses with a mean value of $23 \mu \mathrm{m}$. The density estimates of the chitosan and CNF films are similar, whereas the blends show a clearly sparser structure. The SEM images were taken from the outer side of the films, which might explain the seemingly thinner thicknesses in the images compared to the thickness measurements.
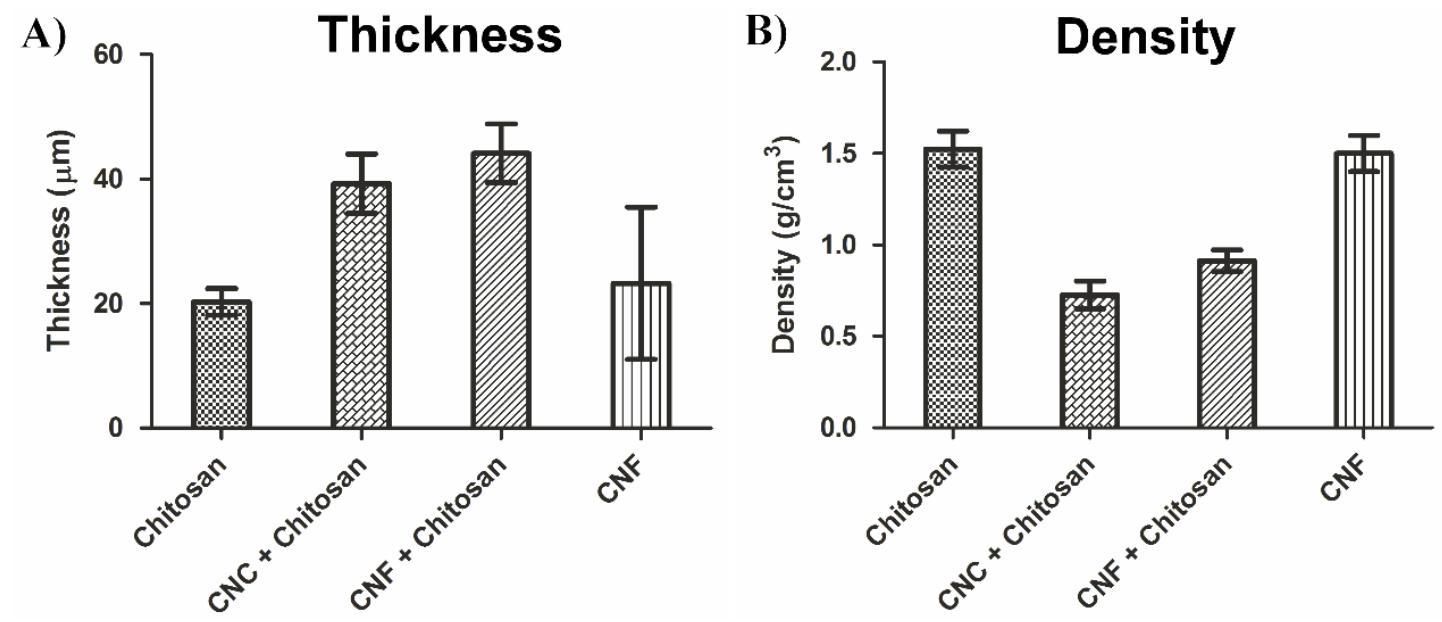
Fig. 3. Mean a) thickness and b) density $(n=7)$ of the solvent casted films. Error bars indicate the standard deviation.

The density of the chitosan film $\left(1.52 \pm 0.10 \mathrm{~g} / \mathrm{cm}^{3}\right)$ is comparable to the literature value of 1.45 g/cm³ (Matsuyama, Kitamura, \& Naramura, 1999; Tsai \& Wan, 2008; Uragami, Kato, \& Miyata, 1997). Correspondingly, the CNF film density $\left(1.50 \pm 0.10 \mathrm{~g} / \mathrm{cm}^{3}\right)$ was only slightly higher than the $1.38 \mathrm{~g} / \mathrm{cm}^{3}$ of the pressure filtered CNF films reported earlier (Rajala et al., 2016). The blend films (CNC-chitosan $0.73 \pm 0.08 \mathrm{~g} / \mathrm{cm}^{3}$; CNF-chitosan $0.91 \pm 0.06 \mathrm{~g} / \mathrm{cm}^{3}$ ) had significantly lower densities compared to the plain chitosan or CNF. The results correlate with the SEM images, which also suggested that the structure of the blends were sparser than plain chitosan or CNF. To conclude, the structure of the plain chitosan and CNF films appeared more organized compared to the blend films.

Thermal stability of the films was evaluated with thermogravimetric analysis. The first thermal event around $100^{\circ} \mathrm{C}$ was denoted dehydration of the samples. Both chitosan and CNF showed a single step main decomposition event. Thermal stability of chitosan was at the same level as reported elsewhere (Sánchez, Alonso, Valencia, \& Franco, 2015) with the main mass loss step occurring at the range from approximately $260{ }^{\circ} \mathrm{C}$ to $320^{\circ} \mathrm{C}$. The corresponding range for $\mathrm{CNF}$ was from $240^{\circ} \mathrm{C}$ to $360^{\circ} \mathrm{C}$. Most of the CNF mass loss occurred after $300^{\circ} \mathrm{C}$, whereas chitosan seemed to decompose in a slightly lower temperature. The blend films decomposed in two partially overlapping stages, caused by the chitosan and nanocellulose components. The two decomposition temperatures are more clearly seen in the case of CNC-chitosan. Both CNC and CNF decomposed in the same temperature range as described previously (Sofla, Brown, Tsuzuki, \& Rainey, 2016). 
a) Chitosan

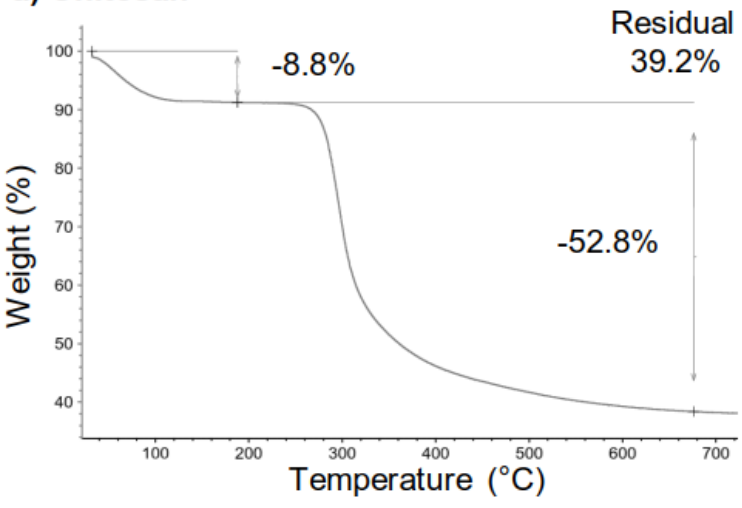

c) CNF + Chitosan

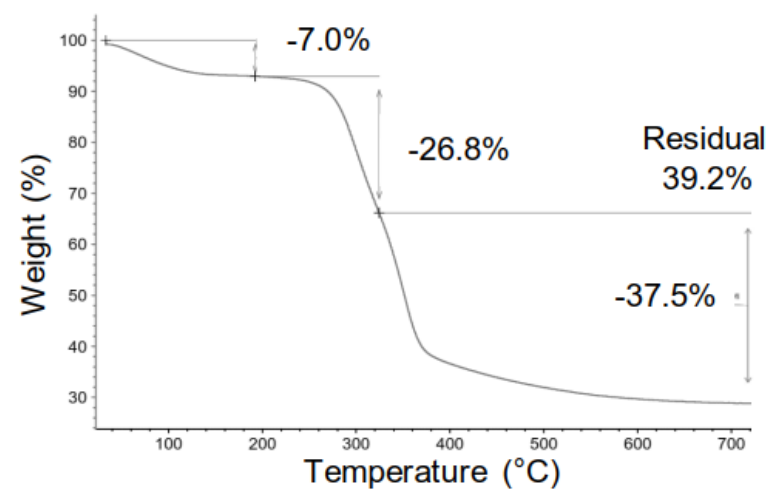

b) CNC + Chitosan

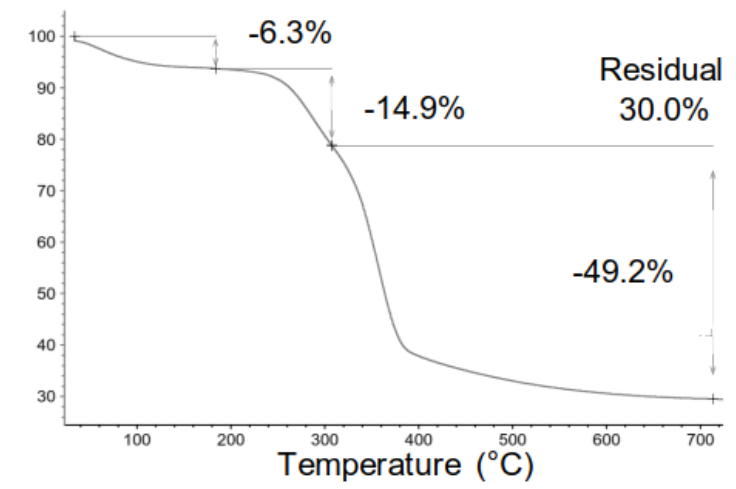

d) CNF

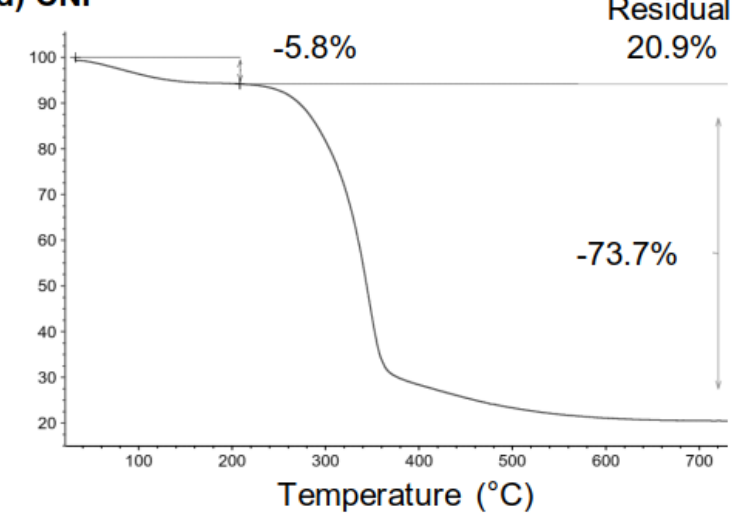

Fig. 4. Thermogravimetric analysis of the solvent casted films.

\subsection{Piezoelectric sensitivity}

Both blend film types had clearly lower piezoelectric sensitivity values $(\sim 2 \mathrm{pC} / \mathrm{N})$ than the plain films of chitosan ( 4-6 pC/N) and CNF ( 7-8 pC/N) as seen in Fig. 5. Thus, the piezoelectric sensitivity of the plain CNF film was in correspondence with our detailed study on CNF films fabricated by pressure filtering (Rajala et al., 2016). The result indicates that the simpler and easily scalable solvent casting method can be used to fabricate piezoelectric CNF membranes without filtration or hot pressing equipment. 


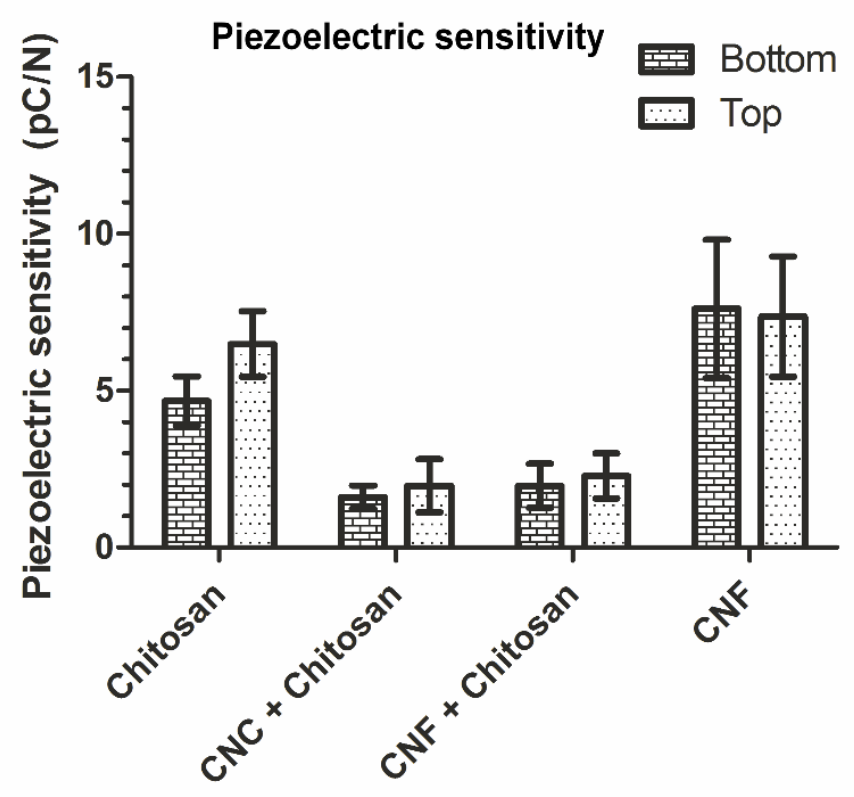

Fig. 5. The mean piezoelectric sensitivities $(n=20)$ and their standard deviations measured from both sides of the solvent casted films.

Previously, adding $15 \%$ of chitosan into collagen films was reported to increase their piezoelectric sensitivity from $0.096 \mathrm{pC} / \mathrm{N}$ to $0.212 \mathrm{pC} / \mathrm{N}$ (Silva et al., 2001). The authors believe that chitosan increased the organization in the microstructure of the films, leading to increased piezoelectricity. Another study reported compressed $1.3 \mathrm{~mm}$ thick chitosan pellets to have a piezoelectric coefficient $\left(d_{33}\right)$ as high as $18.6 \mathrm{pC} / \mathrm{N}$ when using a load of five tons (Praveen et al., 2017). When using a load of one ton, the measured $d_{33}$ value at $300 \mathrm{~K}$ temperature was $4.4 \mathrm{pC} / \mathrm{N}$, which corresponds to the piezoelectric sensitivity of our chitosan film.

In our study, plain chitosan films with piezoelectric sensitivities between 4-6 pC/N were noticed to have more than two times higher values than the $2 \mathrm{pC} / \mathrm{N}$ of quartz crystals (Zhang \& Yu, 2011), demonstrating the potential of microcrystalline chitosan as a biodegradable low cost piezoelectric energy harvesting or sensor material. Furthermore, the antibacterial properties of chitosan make it an attractive alternative for biomedical applications (Kong, Chen, Xing, \& Park, 2010). On the other hand, the higher piezoelectric sensitivity of CNF addresses its suitability for applications where the higher generated charge is essential.

The blend films had a less organized structure and lower piezoelectric sensitivity values compared to the plain chitosan or CNF. We think that disorganization of the nanocellulosechitosan blend films may explain their observed lower piezoelectricity. Hence, studying the effect of aligning the polymer molecules might be useful in improving the piezoelectric sensitivity values of the films. This alignment, or poling, can be achieved for example by utilizing an electrical field across the polymer film (Harrison \& Ounaies, 2002). On the other hand, the effect of the film structure on the measurement should be considered as well; for example surface roughness and film thickness might partly contribute to the measured piezoelectric sensitivity values. 
In contrast to our preliminary study (Hänninen et al., 2016), the blend films had here similar piezoelectric sensitivities regardless whether their top or bottom side was measured. This is in agreement with the apparent structural differences; chitosan and nanocellulose were noticed to form a layered structure in the previous study. The structural differences as well as the different piezoelectric sensitivities between these two studies are expected to arise from the film preparation procedure. As opposed to the earlier study, all the films were now neutralized to a set $\mathrm{pH}$ of 7 before drying them under tension. Moreover, parameters like the humidity and temperature have been shown to affect the properties of solvent casted films (Srinivasa, Ramesh, Kumar, \& Tharanathan, 2004)(Fernández-Pan, Ziani, Pedroza-Islas, \& Maté, 2010)(Suderman, Isa, \& Sarbon, 2016) and should thus be controlled to optimize the film properties.

The piezoelectric chitosan and nanocellulose films could provide biodegradable alternatives for commercial PVDF and EMFi (Paajanen, Lekkala, \& Kirjavainen, 2000) films. Even though the sensitivities of the bio-based films are somewhat lower, they could be suitable for low-cost large-area sensor applications, such as floor sensors.

\subsection{Tensile properties}

The elongation at break describes the ability of a material to resist shape changes without crack formation. The different elongation at break results (Fig. 6b) between CNC-chitosan (2\%) and CNF-chitosan (4\%) blends probably came from the structural differences of cellulose nanocrystals and nanofibers; the longer CNF molecules are more easily entangled than the rod-like highly crystalline CNC whiskers. Due to this same structural feature, solvent casting plain CNC films from the highly fluid dispersion was difficult and no freestanding CNC films were obtained. The easier entanglement of the CNF molecules explains the higher elongation at break and tensile strength in the CNF-chitosan blends compared to CNCchitosan. Plain chitosan films (8\%) were the most flexible as opposed to the plain CNF films $(1 \%)$. 

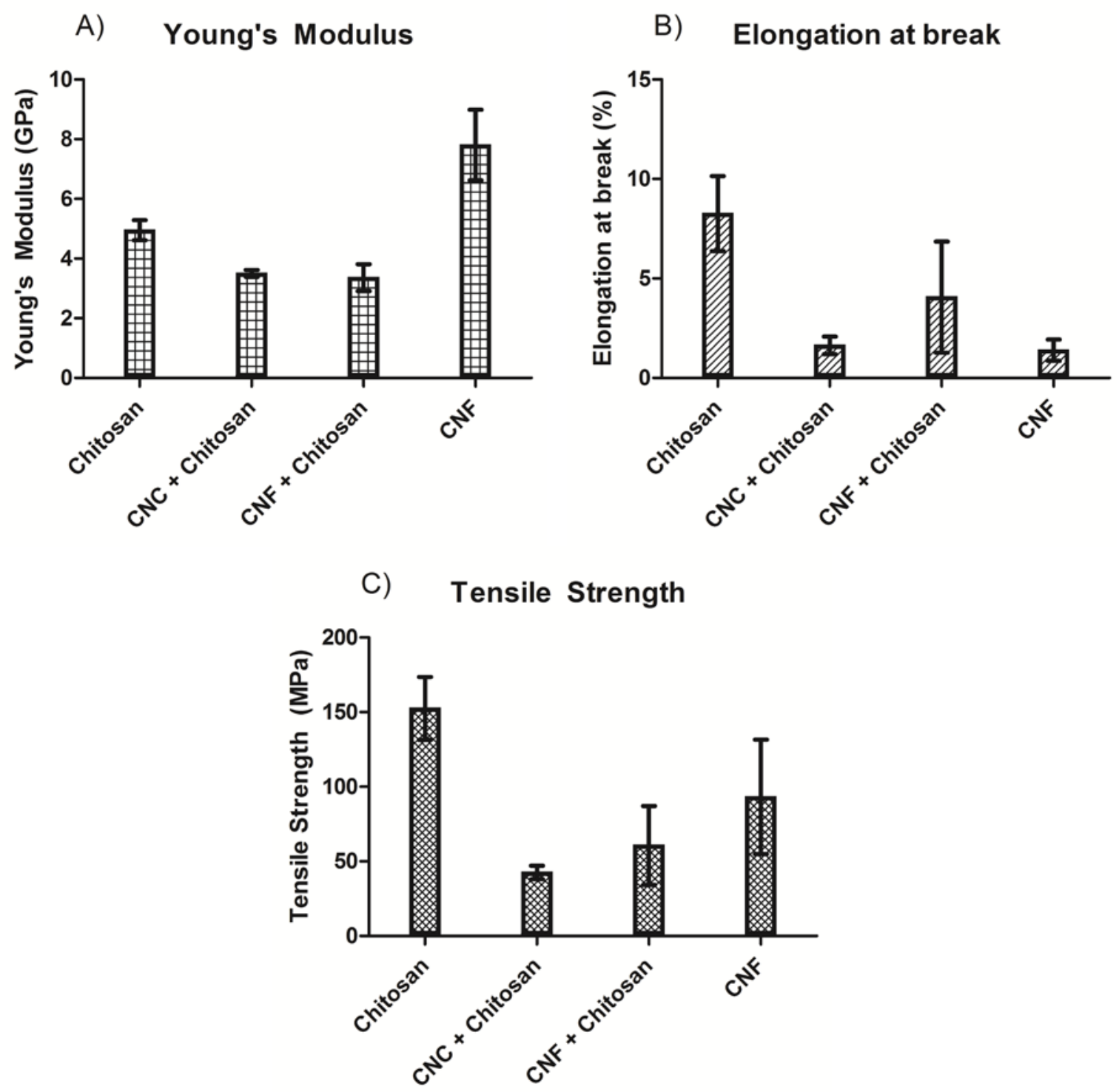

Fig. 6. The tensile properties of the solvent-casted films $(n=3)$. The error bars indicate the standard deviations.

The mechanical properties of different chitosan-nanocellulose blends have been studied extensively in the literature as shown in a recent review paper (Abdul Khalil et al., 2016). In a previous study, low molecular chitosan films were noted to become brittle along with CNF loads of $40-60 \%$, showing elongation at break values of approximately $1-2 \%$ for the blends (Fernandes et al., 2010). Although the results are not directly comparable due to different tensile test parameters, a similar trend was seen(Qian, Jinping, \& Lina, 2009) in our study. This might be attributed to partial deterioration of the bonding between chitosan and nanocellulose, which has been previously reported with increased nanocellulose content as a result of nanocellulose aggregation (Qian et al., 2009).

In comparison to the plain CNF film, the CNF-chitosan blend seemed to have an increased elongation at break. Thus, blending chitosan with CNF is a potential method for decreasing the brittleness of CNF films. Further optimization of the chitosan to nanocellulose ratio could 
be done to adjust the mechanical properties with respect to the piezoelectric sensitivity of the blend films.

Both CNF and CNC have been reported to increase the Young's modulus of chitosan films (Abdul Khalil et al., 2016). As shown in Fig. 6a, in our study both nanocellulose-chitosan blends had a lower modulus (CNC-chitosan 3.5 GPa, CNF-chitosan 3.4 GPa) compared to the chitosan (5.0 GPa) and CNF films (7.8 GPa). The fabrication method is a very likely reason for the poor Young's Modulus and tensile strength of the blends. Evidently, the blend films showed dimensional changes during the second drying phase under tension, as opposed to the chitosan films. Drying the blend films probably led to intrinsic stresses in the films, which could have contributed to their brittle behavior.

The tensile strength of the chitosan film (153 MPa) was higher compared to the CNF film (93 $\mathrm{MPa}$ ). Both blend film types (CNC-chitosan $43 \mathrm{MPa}$, CNF-chitosan $61 \mathrm{MPa}$ ) had lower tensile strength values than the plain polymer films. The entanglement of the longer CNF molecules compared to the CNC probably caused the higher tensile strength of the CNFchitosan blend. The piezoelectricity of chitosan together with its more flexible nature, transparency and higher tensile strength interestingly makes it a potential alternative for CNF for some applications. Considering for example an energy harvesting piezoelectric film embedded in a shoe sole to create sustainable electricity, the film should withstand mechanical stress. Based on the tensile results, chitosan could be a more attractive alternative for such applications compared to the CNF.

\section{Conclusions}

Free-standing biodegradable piezoelectric films were made from chitosan, nanocellulose and their blends. The structure-property relationships of these films were evaluated to find a film type with improved handling durability compared to plain cellulose nanofiber films. In the future, such films could replace non-degradable piezoelectric films for example in powering the Internet of Things. Interestingly, plain chitosan films showed the most promising results with a considerable piezoelectric sensitivity $(4 \mathrm{pC} / \mathrm{N})$ and the highest elongation at break (8\%). As opposed to our hypothesis, the blend films had mostly lower tensile properties compared to the plain films of chitosan and CNF. Only the CNF-chitosan blends were noticed to have an increased elongation at break compared to the plain CNF. Based on the results, orienting the films could be one interesting method for achieving films with better tensile properties and piezoelectric sensitivities. A further research target for overcoming the brittleness of the piezoelectric biopolymers would be to study the effect of plasticizers on the piezoelectric sensitivities of the materials.

\section{Acknowledgements}

The authors would like to thank Mr. Arno Pammo and Mr. Martijn Schouten for performing the piezoelectric sensitivity measurements and Ms. Clara Lessa Belone for performing the thermogravimetric measurements. This research did not receive any specific grant from funding agencies in the public, commercial, or not-for-profit sectors. 


\section{References}

Abdul Khalil, H. P. S., Saurabh, C. K., Adnan, A. S., Nurul Fazita, M. R., Syakir, M. I., Davoudpour, Y., ... Dungani, R. (2016). A review on chitosan-cellulose blends and nanocellulose reinforced chitosan biocomposites: Properties and their applications. Carbohydrate Polymers, 150, 216-226.

Bharimalla, A. K., Deshmukh, S. P., Vigneshwaran, N., Patil, P. G., \& Prasad, V. (2017). Nanocellulose-Polymer Composites for Applications in Food Packaging: Current Status, Future Prospects and Challenges. Polymer-Plastics Technology and Engineering, 56(8), 805-823.

Csoka, L., Hoeger, I. C., Rojas, O. J., Peszlen, I., Pawlak, J. J., \& Peralta, P. N. (2012). Piezoelectric Effect of Cellulose Nanocrystals Thin Films. ACS Macro Letters, 1(7), 867-870.

Ditzel, F. I., Prestes, E., Carvalho, B. M., Demiate, I. M., \& Pinheiro, L. A. (2017). Nanocrystalline cellulose extracted from pine wood and corncob. Carbohydrate Polymers, 157, 1577-1585.

Fernandes, S. C. M., Freire, C. S. R., Silvestre, A. J. D., Pascoal Neto, C., Gandini, A., Berglund, L. A., \& Salmén, L. (2010). Transparent chitosan films reinforced with a high content of nanofibrillated cellulose. Carbohydrate Polymers, 81(2), 394-401.

Fernández-Pan, I., Ziani, K., Pedroza-Islas, R., \& Maté, J. I. (2010). Effect of Drying Conditions on the Mechanical and Barrier Properties of Films Based on Chitosan. Drying Technology, 28(12), 1350-1358.

Frka-Petesic, B., Jean, B., \& Heux, L. (2014). First experimental evidence of a giant permanent electric-dipole moment in cellulose nanocrystals. Europhysics Letters, 107(2), 28006.

Fukada, E. (1955). Piezoelectricity of Wood. Journal of the Physical Society of Japan, 10(2), 149-154.

Fukada, E., \& Sasaki, S. (1975). Piezoelectricity of $\alpha$-chitin. Journal of Polymer Science: Polymer Physics Edition, 13(9), 1845-1847.

Grifoll-Romero, L., Pascual, S., Aragunde, H., Biarnés, X., \& Planas, A. (2018). Chitin Deacetylases: Structures, Specificities, and Biotech Applications. Polymers .

Hänninen, A., Rajala, S., Salpavaara, T., Kellomäki, M., \& Tuukkanen, S. (2016). Nanocrystals, Piezoelectric sensitivity of a layered film of chitosan and cellulose. Eurosensors Conference Proceedings, 168, 1176-1179.

Harrison, J. S., \& Ounaies, Z. (2002). Piezoelectric Polymers. In Encyclopedia of Polymer Science and Technology. John Wiley \& Sons, Inc.

Kong, M., Chen, X. G., Xing, K., \& Park, H. J. (2010). Antimicrobial properties of chitosan and mode of action: A state of the art review. International Journal of Food Microbiology, 144(1), 51-63.

Kumar, M. N. V. R. (2000). A review of chitin and chitosan applications. Reactive and Functional Polymers, 46(1), 1-27. 
Leite, A. L. M. P., Zanon, C. D., \& Menegalli, F. C. (2017). Isolation and characterization of cellulose nanofibers from cassava root bagasse and peelings. Carbohydrate Polymers, 157, 962-970.

Maksimovic, M. (2017). The Role of Green Internet of Things (G-IoT) and Big Data in Making Cities Smarter, Safer and More Sustainable. International Journal of Computing and Digital Systems, 6(4), 175-184.

Matsuyama, H., Kitamura, Y., \& Naramura, Y. (1999). Diffusive permeability of ionic solutes in charged chitosan membrane. Journal of Applied Polymer Science, 72(3), 397404.

Morais, J. P. S., Rosa, M. de F., de Souza Filho, M. de sá M., Nascimento, L. D., do Nascimento, D. M., \& Cassales, A. R. (2013). Extraction and characterization of nanocellulose structures from raw cotton linter. Carbohydrate Polymers, 91(1), 229235.

No, H. K., Meyers, S. P., Prinyawiwatkul, W., \& Xu, Z. (2007). Applications of Chitosan for Improvement of Quality and Shelf Life of Foods: A Review. Journal of Food Science, 72(5), 87-100.

Paajanen, M., Lekkala, J., \& Kirjavainen, K. (2000). ElectroMechanical Film (EMFi) - a new multipurpose electret material. Sensors and Actuators A: Physical, 84(1), 95-102.

Praveen, E., Murugan, S., \& Jayakumar, K. (2017). Investigations on the existence of piezoelectric property of a bio-polymer - chitosan and its application in vibration sensors. RSC Advances, 7(56), 35490-35495.

Qian, L., Jinping, Z., \& Lina, Z. (2009). Structure and properties of the nanocomposite films of chitosan reinforced with cellulose whiskers. Journal of Polymer Science Part B: Polymer Physics, 47(11), 1069-1077.

Rajala, S., Siponkoski, T., Sarlin, E., Mettänen, M., Vuoriluoto, M., Pammo, A., ... Tuukkanen, S. (2016). Cellulose Nanofibril Film as a Piezoelectric Sensor Material. ACS Applied Materials and Interfaces, 8(24), 15607-15614.

Rajala, S., Vuoriluoto, M., Rojas, O. J., Franssila, S., \& Tuukkanen, S. (2015). Piezoelectric sensitivity measurements of cellulose nanofibril sensors. XXI IMEKO 2015 World Congress "Measurement in Research and Industry" Conference Proceedings, (April 2016), 2-6.

Randall, C. A., Kelnberger, A., Yang, G. Y., Eitel, R. E., \& Shrout, T. R. (2005). High Strain Piezoelectric Multilayer Actuators-A Material Science and Engineering Challenge. Journal of Electroceramics, 14(3), 177-191.

Rinaudo, M. (2006). Chitin and chitosan : Properties and applications. Progress in Polymer Science Volume, 31(7), 603-632.

Safari, A., \& Janas, V. F. (1997). Processing of fine-scale piezoelectric ceramic/polymer composites for transducer applications. Ferroelectrics, 196(1), 187-190.

Sánchez, R., Alonso, G., Valencia, C., \& Franco, J. M. (2015). Rheological and TGA study of acylated chitosan gel-like dispersions in castor oil: Influence of acyl substituent and acylation protocol. Chemical Engineering Research and Design, 100, 170-178. 
Silva, C. C., Lima, C. G. A., Pinheiro, A. G., Goes, J. C., Figueiro, S. D., \& Sombra, A. S. B. (2001). On the piezoelectricity of collagen-chitosan films. Physical Chemistry Chemical Physics, 3(18), 4154-4157.

Singh, G., Chandoha-Lee, C., Zhang, W., Renneckar, S., Vikesland, P. J., \& Pruden, A. (2016). Biodegradation of nanocrystalline cellulose by two environmentally-relevant consortia. Water Research, 104, 137-146.

Sofla, M. R. K., Brown, R. J., Tsuzuki, T., \& Rainey, T. J. (2016). A comparison of cellulose nanocrystals and cellulose nano fibres extracted from bagasse using acid and ball milling methods. Advances in Natural Sciences: Nanoscience and Nanotechnology, 7(3), 1-9.

Srinivasa, P. C., Ramesh, M. N., Kumar, K. R., \& Tharanathan, R. N. (2004). Properties of chitosan films prepared under different drying conditions. Journal of Food Engineering, 63(1), 79-85.

Suderman, N., Isa, M. I. N., \& Sarbon, N. M. (2016). Effect of drying temperature on the functional properties of biodegradable CMC-based film for potential food packaging. International Food Research Journal, 23(3), 1075-1084.

Sundaram, J., Pant, J., Goudie, M. J., Mani, S., \& Handa, H. (2016). Antimicrobial and Physicochemical Characterization of Biodegradable, Nitric Oxide-Releasing Nanocellulose-Chitosan Packaging Membranes. Journal of Agricultural and Food Chemistry, 64(25), 5260-5266.

Tomé, L. C., Fernandes, S. C. M., Perez, D. S., Sadocco, P., Silvestre, A. J. D., Neto, C. P., ... Freire, C. S. R. (2013). The role of nanocellulose fibers, starch and chitosan on multipolysaccharide based films. Cellulose, 20(4), 1807-1818.

Tomihata, K., \& Ikada, Y. (1997). In vitro and in vivo degradation of films of chitin and its deacetylated derivatives. Biomaterials, 18(7), 567-575.

Tsai, H., \& Wan, Y. (2008). Properties of hydrophilic chitosan network membranes by introducing binary crosslink agents. Polymer Bulletin, 113(1), 103-113.

Tuukkanen, S., \& Rajala, S. (2015). A survey of printable piezoelectric sensors. 2015 IEEE SENSORS - Proceedings, 1-4.

Uragami, T., Kato, S., \& Miyata, T. (1997). Structure of N-alkyl chitosan membranes on water-permselectivity for aqueous ethanol solutions. Journal of Membrane Science, 124(2), 203-211.

Xu, X., Liu, F., Jiang, L., Zhu, J. Y., Haagenson, D., \& Wiesenborn, D. P. (2013). Cellulose Nanocrystals vs. Cellulose Nanofibrils: A Comparative Study on Their Microstructures and Effects as Polymer Reinforcing Agents. ACS Applied Materials \& Interfaces, 5(8), 2999-3009.

Zhang, S., \& Yu, F. (2011). Piezoelectric Materials for High Temperature Sensors. Journal of the American Ceramic Society, 94(10), 3153-3170. 